\title{
Total thyroidectomy for non-toxic multinodular goiter with versus without the use of harmonic FOCUS dissecting shears - a prospective randomized study
}

\author{
Aleksander Konturek, Marcin Barczyński, Małgorzata Stopa, Wojciech Nowak \\ Department of Endocrine Surgery, $3^{\text {rd }}$ Chair of General Surgery, Jagiellonian University Medical College, Krakow, Poland
}

Videosurgery Miniinv 2012; 7 (4): 268-274

DOI: 10.5114/wiitm.2011.30675

\begin{abstract}
Introduction: Thyroidectomy is among the most commonly performed procedures involving the endocrine glands and the development of advanced surgical methods combined with a strife for performing the operation in a manner that is minimally invasive for the patient has considerably increased the need for instruments that would limit surgical trauma.

Aim: To compare of the outcomes of total thyroidectomy operations with and without the use of ultrasonic harmonic FOCUS dissecting shears.

Material and methods: Eighty-two patients with a bilateral, non-toxic multinodular goiter were randomized to two groups of 41 patients each. Total thyroidectomy was performed in each patient. In the clip-ligation group (CL-G), during thyroidectomy, the superior thyroid vessels were clipped and bipolar coagulation was used to secure smaller vessels, whereas in the harmonic FOCUS group (HF-G), a harmonic device was used to dissect and divide all the thyroid vessels. The statistical analysis included the mean operative time, blood loss, postoperative morbidity and cost-effectiveness.

Results: HF-G vs. CL-G operations were shorter (45.4 \pm 8.7 min vs. $64.5 \pm 14.2 \mathrm{~min} ; p<0.001)$, characterized by a lower mean blood loss $(29.9 \pm 9.8 \mathrm{ml} v \mathrm{vs} .56 .8 \pm 11.0 \mathrm{ml} ; p<0.001)$ and appeared to be more cost-effective $(666.2 \pm 37.5$ EUR vs. $718.0 \pm 69.2$ EUR; $p<0.01)$. No major complications were observed in both groups.

Conclusions: In total thyroidectomy operations, the harmonic FOCUS is safe and facilitates dissection, allowing for a significant decrease in operative time. Other benefits include lower blood loss and a slightly decreased cost of the procedure.
\end{abstract}

Key words: total thyroidectomy, harmonic FOCUS, ultrasonic shears.

\section{Introduction}

Over the past decades, the techniques of thyroid operations have undergone small changes only; however, the last twenty years have seen dynamic development of new instruments that has had a significant impact on the improvement of old and introduction of new surgical techniques [1]. Today, thy- roidectomy is among the most commonly performed procedures involving the endocrine glands and the development of advanced surgical methods combined with the determination to perform the operation in a manner that is minimally invasive for the patient has considerably increased the need for instruments that limit surgical trauma. Appropriate hemostasis and atraumatic tissue dissection in addition to protecting

Address for correspondence:

Aleksander Konturek MD, PhD, Department of Endocrine Surgery, $3^{\text {rd }}$ Chair of General Surgery, Jagiellonian University Medical College, 37 Pradnicka St, 31-202 Krakow, Poland, phone: +48 602661 123, fax: +48 12633 31 05, e-mail: okont@mp.pl 
the tissues against excessive thermal injuries leading to their structural damage have provided foundations for developing an instrument that would combine precision and versatility with safety for the surrounding structures. The ultrasonic knife introduced to operating rooms at the turn of the $20^{\text {th }}$ and $21^{\text {st }}$ centuries has provided new opportunities for safe sealing of blood vessels combined with a minimal thermal effect exerted on the neighboring tissues [2-4]. The operation of a harmonic scalpel based on high-frequency vibrations $(55000 \mathrm{~Hz})$ allows for dissection of blood vessels with simultaneous coagulation and at the same time with minimal lateral thermal tissue damage. Surgical treatment of thyroid diseases is associated with complications, of which bleeding is among the most severe. However, appropriate and cautious surgical techniques combined with exercising care to fully identify anatomical structures have significantly decreased the number of such complications.

\section{Aim}

The objective of the present report was to demonstrate the benefits derived from using a new tip for the ultrasonic harmonic shears (FOCUS) that has been created solely to meet the needs of endocrine surgery in maintaining appropriate intraoperative hemostasis as compared to traditional methods of ligating and thermal coagulation of thyroid vessels.

\section{Material and methods}

\section{Study design and patient selection}

Two hundred and fifty-two patients were referred to the Department of Endocrine Surgery, Third Chair of General Surgery, Jagiellonian University College of Medicine in Krakow, Poland, for thyroid surgery between 09/2010 and 08/2011. Of this group, 119 patients, who were considered for total thyroidectomy for non-toxic multinodular goiter, underwent office high-resolution Doppler ultrasound of the neck with both $7.5 \mathrm{MHz}$ and $12 \mathrm{MHz}$ linear array transducers (Logiq 7; GE, Solingen, Germany) performed by a single endocrine surgeon (MB) with evaluation of the thyroid volume, and distribution and measurements of thyroid nodules. Finally, 82 eligible patients were included in the study. They signed the informed consent form and were randomized with the sealed envelope method $(1: 1)$ to two equal-sized $(n=41)$ groups. The flow diagram of this study is presented in Figure 1. The eligibility criteria included bilateral, non-toxic multinodular goiter, absence of both echographic and biochemical

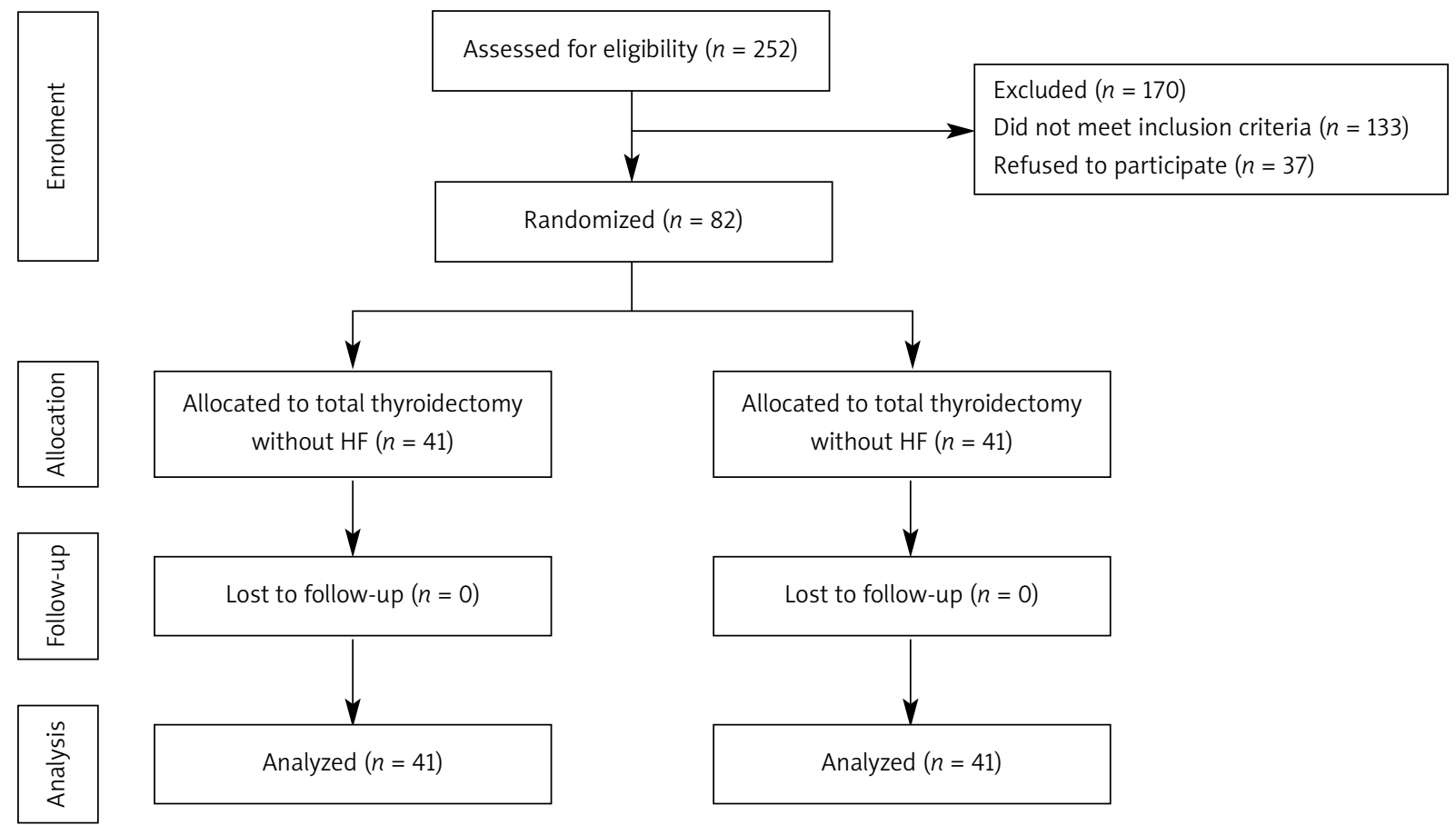

Figure. 1. Flow diagram of the study HF-ultrasonic harmonic FOCUS dissecting shears 
Table I. Characteristics of the two groups of patients who underwent total thyroidectomy with (HF-G) and without (CL-G) Ultrasonic Harmonic FOCUS dissecting shears

\begin{tabular}{|c|c|c|c|}
\hline Parameter & HF-G & CL-G & Value of $p$ \\
\hline Sex (male/female) & $7 / 34$ & $8 / 33$ & $0.72^{\dagger}$ \\
\hline Age [years] & $41.1 \pm 7.5$ & $42.0 \pm 7.5$ & $0.27^{\ddagger}$ \\
\hline Thyroid volume [ml] & $47.5 \pm 17.6$ & $50.9 \pm 15.9$ & $0.44^{\ddagger}$ \\
\hline \multicolumn{4}{|l|}{ Preoperative diagnosis: } \\
\hline $\begin{array}{l}\text { Multinodular non-toxic } \\
\text { goiter }\end{array}$ & 41 & 41 & $1.0^{\dagger}$ \\
\hline
\end{tabular}

Values represent mean \pm standard deviation, unless otherwise indicated. ${ }^{\dagger} \chi^{2}$ test, $\neq t$-test

signs of thyroiditis, no previous neck surgery or neck irradiation, and no suspicion of thyroid malignancy in the fine-needle aspiration biopsy (FNAB) report. The characteristics of the two groups of patients who underwent thyroidectomy with the harmonic FOCUS (HF-G) and without the harmonic FOCUS (CL-G) are presented in Table I. The inclusion criterion was bilateral non-toxic multinodular goiter qualified for firsttime total thyroidectomy. The exclusion criteria included previous thyroid or parathyroid surgery, toxic goiter, FNAB report suspicious of thyroid cancer, history of irradiation, thyroiditis, preoperatively diagnosed recurrent laryngeal nerve palsy, pregnancy or lactation, age below 18 years, high-risk patients ASA (American Society of Anesthesiologists) grade 4, and inability to comply with the scheduled follow-up protocol. In HF-G, the Ultrasonic Harmonic FOCUS device (Ethicon EndoSurgery, Cincinnati, OH, USA) was used to dissect the tissues and to achieve hemostasis, whereas in CL-G, the standard surgical clip-ligation technique for major vessels and bipolar coagulation of minor vessels were used during the operation. The patients were blinded to the relevant group assignment. The primary endpoint of the study was the operating time, whereas the secondary endpoints included blood loss, complications, and cost-effectiveness. The study was approved by the Bioethics Committee of the Jagiellonian University.

\section{Randomization}

Randomization was performed by a computer and sequencing was based on permuted blocks of 3 and 2 to balance the number of patients in the treatment groups. The patients were randomly allocated to one of the treatment groups in a $1: 1$ ratio. Information on the Harmonic FOCUS use remained in consecutively numbered and sealed envelopes that were stored in a specific box in the operating theater. An envelope containing the allocation was added to the patient's file once he had entered the operating room. In this way, the patient was blinded to the relevant group assignment. Then, the envelope was opened and the surgeon performed the assigned intervention.

\section{Anesthesia}

All the total thyroidectomy procedures were performed under general anesthesia. Two anesthesiologists involved in the study followed a strict protocol including premedication with intravenous (IV) midazolam and anesthesia induction with fentanyl, thiopental and pancuronium at a body mass-dependent dose. After the endotracheal intubation, all the patients were put on mechanical ventilation (sevoflurane and oxygen mixture). In the course of the operation, all the patients received an IV infusion of Ringer's solution (15 ml/kg). To prevent postoperative vomiting, IV metoclopramide was administered prior to awakening.

\section{Surgical technique}

All the operations consisted of total extracapsular thyroidectomy and were performed by the same experienced endocrine surgeons involved in the study.

\section{Intraoperative evaluation}

The following parameters were recorded during surgery: operative time (skin incision to skin closure), intraoperative exposure of the recurrent laryngeal nerve and parathyroid glands, blood loss, the number of clips used, the number of vessels coagulated with bipolar coagulation and the number of ligatures used.

\section{Postoperative follow-up}

Neither the patients nor the nurses knew the relevant group assignment. Over-night hospitalization was mandatory for all the patients. For adequate evaluation of biochemical hypocalcemia, blood samples were analyzed in the morning after surgery and on the second postoperative day. Indirect laryngoscopy with a mirror was mandatory on the next day following the operation, being performed by an 
independent ENT specialist to assess recurrent laryngeal nerve morbidity. In cases of recurrent nerve palsy, an additional ENT examination was scheduled at $1,3,6$ and 12 months following surgery.

\section{Cost-effectiveness analysis}

For the cost-effectiveness analysis we used the official in-hospital price list for medical procedures of our hospital (4 EUR per minute of operating theater use, 4 EUR per minute of general anesthesia, 3 EUR per clip, 3 EUR per ligature, 100 EUR for the use of bipolar coagulation), the cost of the Harmonic FOCUS device and the savings in the operating time.

\section{Statistical analysis}

The sample size was estimated based on the principle of detecting a $20 \%$ difference in the operating time with an $80 \%$ probability at $p<0.05$. The statistical significance of categorical variables was evaluated by the $\chi^{2}$ test, whereas the unpaired Student's $t$ test was used for the evaluation of continuous variables (Statistica, Stat-Soft, Katowice). All the data were entered into a dedicated spreadsheet (Microsoft Excel 2002; Microsoft Corporation, San Jose, CA) by a medical assistant and then analyzed by a statistician. Value of $p<0.05$ was considered to indicate significance.

\section{Results}

Eighty-two patients were selected from the above group and divided into two age and goiter size matched groups of 41 subjects each; all these patients underwent total thyroidectomies. Indications for the procedure included multinodular goiter. The results are presented in Table I, while Table II illustrates the intraoperative and follow-up data. The mean operative time in the Harmonic FOCUS group (HF-G) was $45.4 \pm 8.7$ min, ranging from $33 \mathrm{~min}$ to $121 \mathrm{~min}$. In the CL-G group, where clip ligation and bipolar coagulation were employed, the mean operative time was $64.5 \pm 14.2 \mathrm{~min}$, ranging from $45 \mathrm{~min}$ to $137 \mathrm{~min}$. Comparing the mean operative times, the authors observed a statistically significant difference between the groups ( $t$-test: $p<0.001$ ). No significant differences were noted in goiter size or duration of hospitalization. The mean length of skin incision was similar in the two analyzed groups, ranging from $5 \mathrm{~cm}$ to $7 \mathrm{~cm}$. Subsequent assessment focused on the mean postoperative blood loss. In the HF-G group, the value was $29.9 \pm 9.8 \mathrm{ml}$, while the corresponding volume in the $\mathrm{CL}-\mathrm{G}$ group was $56.8 \pm 11.0 \mathrm{ml}$, the difference being statistically significant ( $t$-test: $p<0.001$ ). No early reoperations due to bleeding were noted in the two groups. No significant difference was observed in the risk of transient recurrent laryngeal nerve palsy and hypoparathyroidism. In each group, transient recurrent laryngeal palsy was noted in 1 patient; $4 \mathrm{HF}-\mathrm{G}$ patients and 2 CL-G patients demonstrated a drop in calcium levels below the normal value of $2.0 \mathrm{mmol} / \mathrm{l}$. Cosmetic aspects assessed by a visual analog scale were evaluated 1 month postoperatively. In both roups, the results were similar, the mean score being $82.9 \pm 7.7$ in the HF-G group and $81.9 \pm 5.4$ in the $\mathrm{CL}-\mathrm{G}$ group. The difference was non-significant ( $t$-test; $p<0.001)$. The total cost of the procedure performed employing the harmonic shears was lower (adjusted for Polish conditions); when converted from Polish zlotys to EUR, it was $666.2 \pm 37.5$ EUR in the HF-G group and 718.0 \pm 69.2 EUR in the CL-G group $(p<0.01)$.

\section{Discussion}

The thyroid is one of the largest endocrine glands in humans. Blood flow through the gland is approximately $8 \mathrm{ml} / 100 \mathrm{~g} / \mathrm{s}$ and it may double in hyperthyroid states (16-17 $\mathrm{ml} / 100 \mathrm{~g}$ tissue/s). Thus, the thyroid is the best blood-supplied organ in our body. The development of operative techniques in endocrine

Table II. Intraoperative and follow-up data

\begin{tabular}{|c|c|c|c|}
\hline Parameter & $\mathrm{HF}-\mathrm{G}$ & $\mathrm{CL}-\mathrm{G}$ & Value of $p$ \\
\hline Operative time [min] & $45.4 \pm 8.7$ & $64.5 \pm 14.2$ & $<0.001^{\dagger}$ \\
\hline Blood loss $[\mathrm{ml}]$ & $29.9 \pm 9.8$ & $56.8 \pm 11.0$ & $<0.01^{\dagger}$ \\
\hline Clips/ligatures used (n) & $1 / 0$ & $3 \pm 1 / 31 \pm 5$ & $<0.001^{\ddagger}$ \\
\hline $\begin{array}{l}\text { Vessels secured with } \\
\mathrm{BC}(n)^{\star}\end{array}$ & 0 & $32 \pm 12$ & $<0.001^{\ddagger}$ \\
\hline \multicolumn{4}{|l|}{ Complications (n): } \\
\hline Wound seroma & 0 & 1 & $0.31^{\dagger}$ \\
\hline Transient hypocalcemia & 4 & 2 & $0.55^{\dagger}$ \\
\hline Transient RLN paresis & 1 & 1 & $0.31^{\dagger}$ \\
\hline Postoperative stay [days] & $1.3 \pm 0.5$ & $1.4 \pm 0.6$ & $0.51^{\ddagger}$ \\
\hline \multicolumn{4}{|c|}{$\begin{array}{l}\text { Costs of the operations } 666.2 \pm 32.1718 .0 \pm 27.2 \\
\text { [EUR] }\end{array}$} \\
\hline
\end{tabular}

Values represent mean + standard deviation, unless otherwise indicated. $B C$ - bipolar coagulation, VAS - visual analogue scale (points), RLN-recurrent laryngeal nerve. ${ }^{\dagger} \chi^{2}$ test, ${ }^{\ddagger}$ t-test 
surgery, and especially in thyroid surgery, was for many years limited by the need to achieve appropriate hemostasis. Intraoperative and postoperative bleeding frequently led to serious consequences, from immediate reoperation to severe damage of vitally important structures, such as laryngeal nerves and parathyroids. Such complications were predominantly the consequence of inappropriate identification of thyroid-surrounding tissues and lack of care to perform atraumatic tissue dissection. Traditional thyroidectomy based on blood vessel ligation using ligatures or vascular clips became a time-consuming procedure, associated with a risk of postoperative bleeding. Unfortunately, the technique also restricted the development of minimally invasive operations, where pain reduction and good cosmetic effects were achieved. Procedures performed in a small surgical field were limited by technical capabilities. The introduction of bipolar pincers allowed for safe coagulation of small blood vessels; nevertheless, surgery continued to pose a grave risk of lateral thermal damage of the nerves and parathyroids while dissecting the thyroid.

At the end of the $20^{\text {th }}$ century, a new device was introduced to operating theatres, namely the ultrasonic scalpel, which ideally reconciled the need of appropriate hemostasis with atraumatic tissue dissection. The effect exerted by the shears on the tissue can be basically divided into three periods, depending on three different physico-chemical phenomena, namely coaptation, cavitation and coagulation, occurring with the temperature curve increasing in time. This allowed for sealing blood vessels with a diameter as small as $5 \mathrm{~mm}$ without a need for additional protection by ligation, thus significantly shortening operative time and decreasing the use of material (sutures, clips). The first complete report based on randomized studies on the use of the ultrasonic shears in thyroid surgery was prepared by Voutilainen et al., based on a small group of patients. Of 36 individuals, the authors selected 19 and subjected them to procedures performed using the harmonic scalpel, noting a mean decrease of operative time by $35 \mathrm{~min}$. The results were concordant with other publications presenting randomized studies of surgical patients $[5,6,8,9]$. A decreased operative time both for unilateral lobectomies and for minimally invasive videoassisted total thyroidectomies (MIVAT) was also observed by the team headed by Professor P. Miccoli. Initially, the too small group of patients in whom the ultrasonic shears were employed (only 26 of 116 individuals included in the study) did not allow for a firm opinion on the advantages and disadvantages of the new instrument [2]. Nevertheless, the subsequent reports published by Professor Miccoli's team (833 patients) unambiguously confirmed a significant decrease in operative time to the mean value of 36 min for lobectomies and 46 min for thyroidectomies, combined at the same time with a low rate of permanent complications: unilateral palsy of the recurrent laryngeal nerve in 7 patients $(0.8 \%)$ and fixed hypoparathyroidism in 2 individuals (0.3\%) [3].

The use of the ultrasonic scalpel significantly decreased blood loss. Numerous randomized trials that focused both on open technique and on videoassisted procedures performed in simple and hyperthyroid goiter (Graves disease) reported a decrease of intraoperative blood loss with a simultaneous reduction of postoperative drainage $[4,7,9,12,16,21]$.

The problem of permanent paralysis of vocal cords and hypoparathyroidism occurring in procedures performed using the ultrasonic scalpel was reflected in numerous publications. The reports of Marcesi et al. and Voutilainen et al. and the commentary written by Professor Dionigi [10, 22, 28] presented the dangers and consequences resulting from using the instrument, whose thermal effect on the laryngeal nerves and parathyroid glands had not been fully elucidated. The papers emphasized an increased incidence of such complications, which resulted from the lateral thermal effect of the scalpel tip on vitally important structures. The above-mentioned concerns were not supported by numerous randomized studies [16, 19, 20, 21], the authors of which stressed that although the new instrument was very useful in tissue preparation and dissection with simultaneous coagulation, the surgeon was not released from the obligation to correctly and safely identify the surrounding tissues; nor was he allowed to carelessly use the new shears in the close vicinity of the nerves and parathyroid glands. Incidentally, the above words of caution should not refer only to the harmonic scalpel, but also to traditional methods of vessel coagulation [23-25].

The new tip of the ultrasonic shears - HARMONIC FOCUS - introduced in 2008 is a new generation instrument; its curved tip allows for simultaneous dissection, coagulation and division of tissue structures, at the same time making it possible to safely seal blood vessels of a diameter as small as $5 \mathrm{~mm}$. 
The surgical experience acquired to date in using the ultrasonic scalpel (HS CS-14C and HS ACE-14S tips) $[9,16]$ and the proven benefits, presented in the literature, resulting from using the instrument prompted the present authors to introduce the new generation ultrasonic scalpel while performing open thyroidectomies. The above quoted data derived from the literature on the currently used harmonic scalpel tips were fully confirmed in the results of the study of a randomized group of patients. The skills and experience acquired to date by the surgical team in using the ultrasonic shears have markedly facilitated the use of the Harmonic Focus tip in nodular goiter surgery.

It should be emphasized that in the two investigated groups of patients, both the operative time and blood loss were statistically lower in the Harmonic Focus group. Both the thyroid volume and the character of nodular lesions in the thyroid parenchyma of the 2 patient groups were comparable. A decreased operative time was observed in the HF-G group, the drop amounting to approximately $30 \%$ as compared to the CL-G group. At the same time, intraoperative blood loss decreased by approximately $47 \%$. No statistical differences were noted in the number and quality of postoperative complications. Postoperative drainage was not employed routinely in all the patients; only a single patient demonstrated subcutaneous edema combined with exudate, which was drained postoperatively. No statistical inter-group differences in duration of hospitalization were noted. While analyzing economic benefits in particular groups, the authors observed a decrease in the total cost of the procedure of total thyroidectomy in patients with nodular goiter operated on using the ultrasonic scalpel. The mean total cost of the procedure (expressed in EUR and adjusted for Polish conditions) was lower by approximately 50-60 EUR in patients operated on using the ultrasonic shears. In the opinion of the present authors, both the reduction of operative time and lower (however slightly) costs of the procedure combined with a better use of the operating theater would significantly contribute to shortening the surgery waiting list, at the same time reducing the costs of operation. Very similar conclusions were presented in other publications on the subject. The reports of Ortega et al., Barczyński et al., and Lombardi et al. [5, 16, 19] that focused on randomized groups of patients treated surgically for thyroid diseases not only demonstrated the above difference in operational time, but also a real decrease of the total costs of hospitalization.

In summary, the harmonic scalpel has become an instrument that can be used in all soft tissue procedures. It has allowed for simultaneous hemostasis, tissue preparation and a low-temperature effect on adjacent structures. More than 10 years of experience in employing the scalpel have provided us with strong arguments for advocating its wide use. Its effectiveness has been unambiguously proven in thyroid surgery, both in minimally invasive (video-assisted) and in open thyroidectomies.

\section{Conclusions}

In total thyroidectomy operations, the harmonic FOCUS is safe and facilitates dissection, allowing for a significant decrease in operative time. Other benefits include lower blood loss and a slightly decreased cost of the procedure.

\section{References}

1. Becker AM, Gourin CG. New technologies in thyroid surgery. Surg Oncol Clin N Am 2008; 17: 233-48.

2. Miccoli P, Berti P, Raffaelli M et al. Impact of harmonic scalpel on operative time during video-assisted thyroidectomy. Surg Endosc 2002; 16: 663-6.

3. Miccoli P, Berti P, Frustaci GL, et al. Video-assisted thyroidectomy: indications and results. Langenbecks Arch Surg 2006; 391: 68-71.

4. Defechereux T, Rinken F, Maweja S et al. Evaluation of the ultrasonic dissector in thyroid surgery. A prospective randomized study. Acta Chir Belg 2003; 103: 274-7.

5. Ortega J, Sala C, Flor B, Lledo S. Efficacy and cost-effectiveness of the UltraCision harmonic scalpel in thyroid surgery: an analysis of 200 cases in a randomized trial. J Laparoendosc Surg Tech A 2004; 14: 9-12.

6. Cordon C, Fajardo R, Ramirez J, et al. A randomized, prospective, parallel group study comparing the harmonic scalpel to electocautery in thyroidectomy. Surgery 2005; 137: 337-41.

7. Kilic M, Keskek M, Ertan T, et al. A prospective randomized trial comparing the harmonic scalpel with conventional knot tying thyroidectomy. Adv Ther 2007; 24: 632-8.

8. Manouras A, Markogiannakis H, Koutras AS, et al. Thyroid surgery: comparison between the electrothermal bipolar vessel sealing system, harmonic scalpel, and classic suture ligation. Am J Surg 2008; 195: 48-52.

9. Konturek A, Barczynski M, Cichon S, et al. Ultrasonic scalpel versus classic procedures with limited access in thyroid gland surgery. Pol J Surg 2007; 79: 848-59.

10. Shemen L. Thyroidectomy using the harmonic scalpel: Analysis of 105 consecutive cases. Otolaryngol Head Neck Surg 2002; 127: 284-8.

11. Coimbra C, Defechereux T, Meurisse N, et al. Ultrasonic scalpel in thyroid surgery. Rev Med Liege 2007; 62: 83-5. 
12. Defechereux T, Meurisse M. Hemostasis and ultrasonic shears for thyroid surgery. Ann Chir 2006; 131: 154-6.

13. Siperstein AE, Berber E, Morkoyun E. The use of the harmonic scalpel vs. conventional knot tying for vessel ligation in thyroid surgery. Arch Surg 2002; 137: 137-42.

14. Kanehira E, Omura K, Kinoshita T. How secure are the arteries occluded by a newly developed ultrasonically activated device? Surg Endosc 1999; 13: 340-2.

15. Harold KL, Pollinger $\mathrm{H}$, Matthews BD, et al. Comparison of ultrasonic energy, bipolar thermal energy, and vascular clips for the hemostasis of small-, medium-, and large-sized arteries. Surg Endosc 2003; 17: 1228-30.

16. Barczyński M, Konturek A, Cichoń S. Minimally invasive videoassisted thyreoidectomy (MIVAT) with and without use of harmonic scalpel: a randomized study. Langenbecks Arch Surg 2008; 393: 647-54.

17. Yildirim O, Umit T, Ebru M, et al. Ultrasonic harmonic scalpel in total thyroidectomies. Adv Ther 2008; 25: 260-5.

18. Leonard DS, Timon C. Prospective trial of the ultrasonic dissector in thyroid surgery. Head Neck 2008; 30: 904-8.

19. Lombardi CP, Raffaelli M, Cicchetti A, et al. The use of "harmonic scalpel" versus "knot tying" for conventional "open" thyroidectomy: results of a prospective randomized study. Langenbecks Arch Surg 2008; 393: 627-31.

20. Sartori PV, De Fina S, Colombo G, et al. Ligasure versus Ultracision in thyroid surgery: a prospective randomized study. Langenbecks Arch Surg 2008; 393: 655-8.

21. Hallgrimsson P, Lovén L, Westerdahl J, et al. Use of the harmonic scalpel versus conventional haemostatic techniques in patients with Grave disease undergoing total thyroidectomy: a prospective randomised controlled trial. Langenbecks Arch Surg 2008; 393: 675-80.

22. Dionigi G. Energy based devices and recurrent laryngeal nerve injury: the need for safer instruments. Langenbecks Arch Surg 2009; 394: 579-80.

23. Barczyński M. Reply to G. Dionigi's letter: energy based devices and recurrent laryngeal nerve injury: the need for safer instruments. Langenbecks Arch Surg 2009; 394: 581-2.

24. Lombardi CP, Raffaelli M, Cicchetti A, et al. Reply to G. Dionigi's letter: energy based devices and recurrent laryngeal nerve injury: the need for safer instruments. Langenbecks Arch Surg 2009; 394: 583-4.

25. Sartori PV, Colombo G, Pugliese F, et al. Reply to G. Dionigi's letter: energy based devices and recurrent laryngeal nerve injury: the need for safer instruments. Langenbecks Arch Surg 2009; 394: 585-6.

26. Prgomet D, Janjanin S, Bilić M, et al. A prospective observational study of 363 cases operated with three different harmonic scalpels. Eur Arch Otorhinolaryngol 2009; 266: 1965-70.

27. Manouras A, Markogiannakis HE, Kekis PB, et al. Novel hemostatic devices in thyroid surgery: electrothermal bipolar vessel sealing system and harmonic scalpel. Expert Rev Med Devices 2008; 5: 447-66.

28. Voutilainen PE, Haglund $\mathrm{CH}$. Ultrasonically activated shears in thyroidectomies - a randomized trial. Ann Surg 2000; 231: 322-32.

Received: 1.03.2012, Revised: 23.04.2012, Accepted: 27.05.2012. 